\title{
Anisotropic Fluid Phase Formed by Ionic Liquid-Crystalline Materials with Ammonium lons
}

\author{
Shiori Tomitaka, Jinzhi Wu, Masanori Nata and Seiji Ujiie*
}

Department of Applied Chemistry, Faculty of Engineering, Oita University, 700 Dannoharu, Oita 870-1192, Japan

\begin{abstract}
Ionic liquid-crystalline materials (ILCMs) with ammonium ions were synthesized. Their thermal and orientational properties were studied by polarizing microscopy, differential scanning calorimetry, and X-ray diffraction (XRD). The ILCMs formed the smectic A phase on heating and cooling. A focal conic fan texture was observed in the phase. In addition, the ILCMs spontaneously formed a perpendicular alignment in the smectic A phase. The XRD patterns of the ILCMs consisted of sharp reflections in the small-angle region and broad band in the wide-angle region in the smectic A phase.
\end{abstract}

Keywords: Anisotropic Fluid, Ionic Liquid Crystal, Thermal Stability, X-ray diffraction.

\section{INTRODUCTION}

Most liquid-crystalline materials are neutral organic compounds. The driving forces for the formation of the liquid crystals are interactions between the anisometric molecules such as dipole-dipole interactions, van der Waals interactions, and $\pi-\pi$ stacking. Ionic liquidcrystalline materials (ILCMs), which are a class of liquid-crystalline compounds that contain anions and cations, can be considered as materials that combine the properties of liquid crystals and ionic liquids [1-3]. Owing to the ionic character, some of the properties of the ionic liquid crystals (ILCs) differ significantly from those of conventional liquid crystals. The ionic interactions usually tend to stabilize lamellar mesophases. However, ionic liquid crystals can also display uncommon mesophases like the nematic columnar phase $[4,5]$.

Ammonium salts with long alkyl chains are wellknown cationic surfactants and are effective surface treatment agents because of the formation of a perpendicular alignment. In our previous work, we reported the formation of ionic liquid crystals with a nitro terminal group $[2,6]$. In these ionic liquid crystals, alkylammonium, piperidinium, and imidazolium cations were used as ionic skeletons. It was reported that the ILCM with a hexamethylene spacer chain and a butoxy terminal group forms the smectic A phase [3]. In this work, ILCs with different spacer chains, belonging to the family of ILCMs, were synthesized. The thermal and orientational properties of the ILCs have been described. The ionic interactions in the

${ }^{*}$ Correspondence Address to this author at the Department of Applied Chemistry, Faculty of Engineering, Oita University, 700 Dannoharu, Oita 8701192, Japan; Tel/Fax: +81-97-554-7903; E-mail: seujiie@oita-u.ac.jp
ILCs were found to be effective in forming a thermotropic liquid crystalline phase with enhanced thermal stability.

\section{METHODS}

\subsection{Measurements}

The proton nuclear magnetic resonance $\left({ }^{1} \mathrm{H}\right.$ NMR; $400 \mathrm{MHz}$ ) spectra were determined using a Bruker AV400 NMR spectrometer. The phase transitions were measured using a Mettler TA-60Ws system, a Shimadzu DSC60, and a Nikon polarizing microscope with a Mettler FP82HT hot stage apparatus attached with a Mettler FP90 controller. The X-ray diffraction (XRD) studies were performed using a Shimadzu X-ray Rad-6100 system (Ni-filtered CuKa radiation).
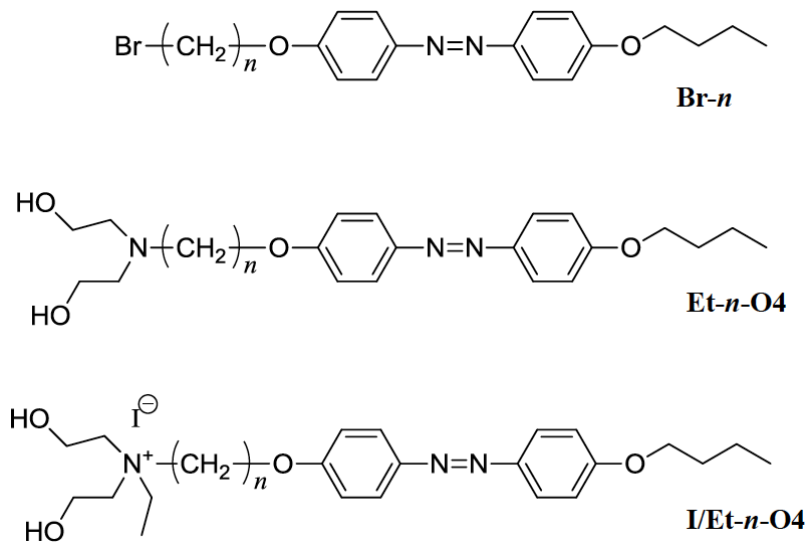

Figure 1: Structures of $\mathrm{Br}-n, \mathrm{Et}-n-\mathrm{O} 4$ and $\mathrm{l} / \mathrm{Et}-n-\mathrm{O} 4(n=3,6$, 7 , and 11).

\subsection{Synthesis}

\section{4-(4-(butoxy)phenylazo)phenyl (AzoBu)}

4- $n$-butoxyaniline $(9.55 \mathrm{~g}, 57.8 \mathrm{mmol})$ was dissolved in hydrochloric acid $(35 \%, 20 \mathrm{~mL})$, and the solution was 
cooled below $5{ }^{\circ} \mathrm{C}$. For the diazotization, sodium nitrite $(4.02 \mathrm{~g}, 58.3 \mathrm{mmol})$ was added to the solution at a temperature below $5{ }^{\circ} \mathrm{C}$. Subsequently, a mixture of sodium hydroxide solution with phenol $(5.60 \mathrm{~g}, 59.5$ $\mathrm{mmol}$ ) was added to the cooled solution. The reaction mixture was stirred for $1 \mathrm{~h}$ at about $5{ }^{\circ} \mathrm{C}$ and then the reaction was acidified using concentrated hydrochloric acid. The precipitate was isolated by filtration, washed with water until the $\mathrm{pH}$ of the filtrate reached 7 , and then dried. The yield of AzoBu was $86.9 \%$.

\section{7-bromo-1-(4-(4- \\ (butoxy)phenylazo)phenoxy)heptane (Br-7)}

AzoBu (1.00 g, $3.70 \mathrm{mmol})$, 1,7-dibromoheptane $(5.84 \mathrm{~g}, 22.64 \mathrm{mmol})$, and anhydrous potassium carbonate $(2.76 \mathrm{~g}, 19.97 \mathrm{mmol})$ were added to acetone $(400 \mathrm{~mL})$, and the reaction mixture was heated to 75 ${ }^{\circ} \mathrm{C}$ and refluxed for $24 \mathrm{~h}$. After the reaction, the hot solution was immediately filtered off and the inorganic residues were washed thoroughly with hot acetone. The filtrates were collected and evaporated under vacuum to remove the acetone. The precipitated product was filtered off and recrystallized from hexane to yield $1.22 \mathrm{~g}$ of orange crystals. The yield of $\mathrm{Br}-7$ was $73.5 \%$.

\section{1-(bis(2-hydroxyethyl)amino)-7-(4-(4- (butoxy)phenylazo)phenoxy)heptane (Et-7-04)}

Br-7 $(0.40 \mathrm{~g}, 0.89 \mathrm{mmol})$, diethanolamine $(0.10 \mathrm{~g}$, $0.99 \mathrm{mmol})$ and anhydrous potassium carbonate $(0.14$ $\mathrm{g}, 0.99 \mathrm{mmol}$ ) were added to 2-propanol (100 $\mathrm{mL})$ under magnetic stirring, and the reaction mixture was stirred at $120^{\circ} \mathrm{C}$ for $58 \mathrm{~h}$. After the reaction, the mixed solution was evaporated under vacuum, and the residues were dissolved in chloroform. The resulting chloroform solution was added to distilled water in a separating funnel and shaken vigorously to remove any excess amounts of diethanolamine and $\mathrm{KBr}$ generated during the reaction, until the water phase became neutral. The organic chloroform layer was dried over anhydrous magnesium sulfate to remove any water remaining in the solution. After removing the solvent by reduced pressure distillation, the solution was concentrated in hexane $(\mathrm{v} / \mathrm{v}=1 / 10)$. The target material precipitated from the solution and was filtered off. The solution was dried under vacuum to yield 0.19 $\mathrm{g}$ of yellow crystalline powder. The yield of Et-7-O4 was $47.4 \%$.

\section{Bis(2-hydroxyethyl)ethyl(7-(4-(4- (butoxy)phenyazo)phenoxy)heptyl)ammonium iodide (I/Et-7-04)}

Et-7-O4 $(0.15 \mathrm{~g}, 0.37 \mathrm{mmol})$ and iodoethane $(0.08$ $\mathrm{g}, 0.51 \mathrm{mmol})$ were dissolved in 2-propanol $(30 \mathrm{~mL})$, and the mixed solution was reacted at $130{ }^{\circ} \mathrm{C}$ for $49 \mathrm{~h}$. After the reaction, the mixed solution was condensed by evaporating under vacuum. The precipitation (I/Et-6O4) was filtered off. The yield of I/Et-7-O4 was $47.6 \%$.

${ }^{1} \mathrm{H}$ NMR $\left(\mathrm{CDCl}_{3}, 400 \mathrm{MHz}\right) \delta(\mathrm{ppm}): 0.98(3 \mathrm{H}$, $\left.\mathrm{CH}_{3}\right), 1.32-1.56\left(11 \mathrm{H},-\mathrm{CH}_{2^{-}}, \mathrm{CH}_{3}-\right), 1.74-1.82(6 \mathrm{H}$, $\left.\mathrm{CH}_{2}-\right), 2.50(2 \mathrm{H},-\mathrm{OH}), 3.08-3.69\left(8 \mathrm{H},-\mathrm{CH}_{2}-\mathrm{N}^{+}\right), 3.95-$ $4.01\left(8 \mathrm{H}, \mathrm{HO}-\mathrm{CH}_{2^{-}}, \mathrm{CH}_{2}-\mathrm{O}-\right.$-phenyl), $6.98(4 \mathrm{H}$, phenyl), $7.85(4 \mathrm{H}$, phenyl). The ILCs of I/Et-3-O4 and I/Et-11-O4 were synthesized by the same method.

\section{RESULTS AND DISCUSSION}

\subsection{Phase Transitions}

The phase transitions of $\mathrm{Br}-n$ and $\mathrm{Et}-n-\mathrm{O} 4$, where $n$ $=3,6,7$, and 11 are summarized in Table 1. The phase transitions of Et-6-O4 and I/Et-6-O4 have been reported in the literature [3]. $\mathrm{Br}-3$ did not exhibit a thermotropic liquid-crystalline phase whereas $\mathrm{Br}-6$ showed a nematic phase. Both $\mathrm{Br}-7$ and $\mathrm{Br}-11$ showed the smectic $A$ and the nematic phases. Et-3-O4, Et-6O4, Et-7-O4, and Et-11-O4 did not form thermotropic liquid-crystalline phases on heating, whereas Et-11-O4 showed a nematic phase on cooling.

The phase transitions of I/Et-n-O4 $(n=3,6,7$, and $11)$, obtained by the reaction of Et-n-O4 with ethyl

Table 1: Phase Transitions of Br-n, Et-n-O4, and I/Et-n-O4 ( $n=3,6,7$, and 11)

\begin{tabular}{|c|c|c|c|}
\hline \multirow{2}{*}{$\mathbf{n}$} & \multicolumn{3}{|c|}{ Phase Transition Temperatures $1 /{ }^{\circ} \mathrm{C}$} \\
\hline & $B r-n$ & Et-n-O4 & I/Et-n-04 \\
\hline 3 & k 117.1 I & k 128.0 I & k 108.7 SmA 139.9 I \\
\hline $6^{2}$ & k 99.7 N 113.9 I & k 110.5 I & k 117.8 SmA 169.7 I \\
\hline 7 & k 86.8 SmA 103.8 N 113.0 I & k 110.9 I & k 119.7 SmA 176.8 I \\
\hline 11 & k 78.4 SmA 90.2 N 103.7 I & k 112.4 I & k 112.8 SmA 151.2 I \\
\hline
\end{tabular}

${ }^{1} \mathrm{k}$ : solid, SmA: smectic $\mathrm{A}, \mathrm{N}$ : nematic, I: isotropic.

${ }^{2}$ Phase transition temperatures of Et-6-O4 and I/Et-6-O4 were reported in Ref. [3] 


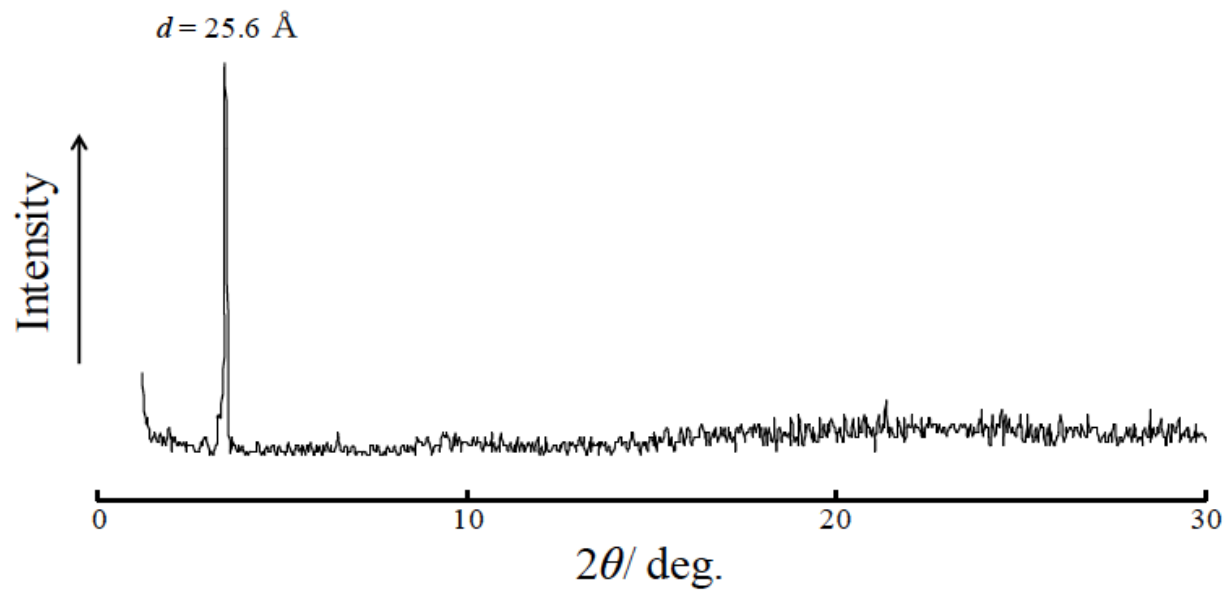

Figure 2: XRD pattern of $\mathrm{Br}-7$ at $90^{\circ} \mathrm{C}$.

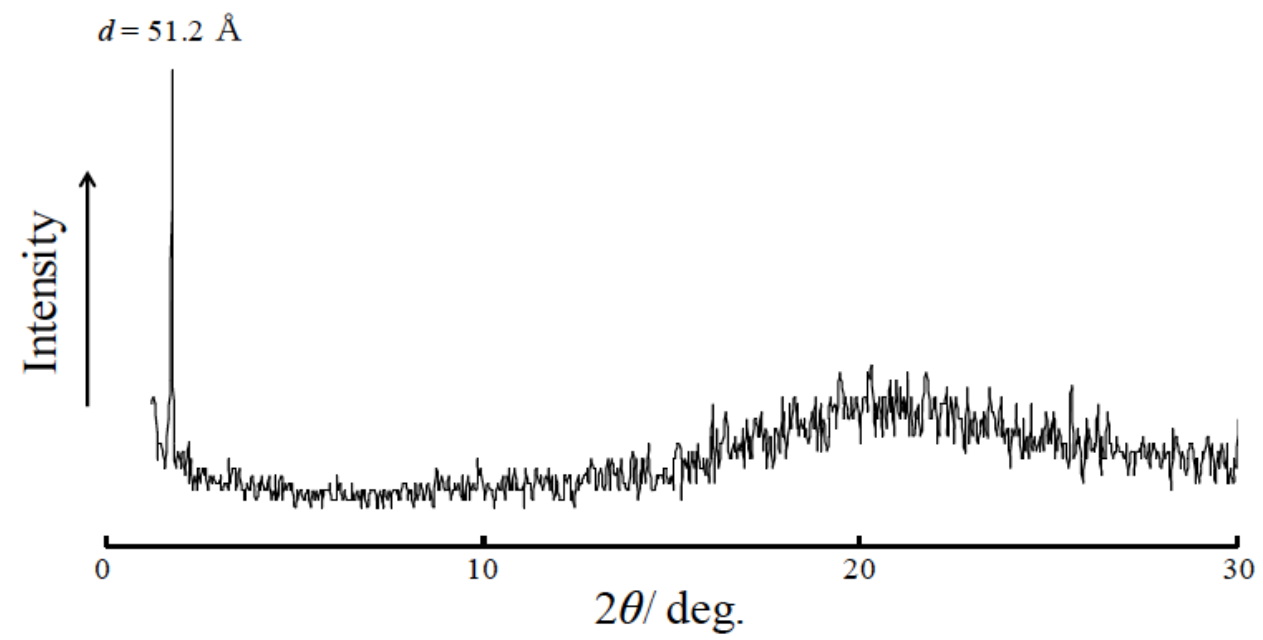

Figure 3: XRD pattern of I/Et-7-O4 at $130^{\circ} \mathrm{C}$.

iodide, are also summarized in Table 1. Et- $n$-O4 did not form a thermotropic liquid-crystalline phase. However, I/Et- $n$-O4 showed the smectic A phase both on heating and cooling. Furthermore, I/Et- $n$-O4 exhibited a higher isotropization temperature than Et-n-O4. The smectic A phase with enhanced thermal stability was formed due to the ionic interactions between the ammonium and the iodide ions in I/Et-n-O4. Similar results have been reported in previous papers $[6,9]$.

\subsection{Orientational Properties}

The XRD patterns of $\mathrm{Br}-7$ and $\mathrm{I} / \mathrm{Et}-7-\mathrm{O} 4$ are shown in Figures 2 and 3, respectively. The possible packing models of $\mathrm{Br}-7$ and I/Et-7-O4 are presented in Figure 4. The first-order reflection in the small-angle region of the XRD pattern of $\mathrm{Br}-7$ corresponds to a layer spacing $(d)$ of $25.6 \AA$ at $90{ }^{\circ} \mathrm{C}$ while the extended molecular length $(L)$ of $\mathrm{Br}-7$ is $24.8 \AA$. The value of $d$ is approximately equal to that of $L$, indicating the formation of a monolayer structure of the smectic A phase. The firstorder diffraction peak in the small-angle region of the XRD pattern of I/Et-7-O4 corresponds to a layer spacing of $51.2 \AA$ at $130{ }^{\circ} \mathrm{C}$, while the extended molecular length of I/Et-7-O4 is $30.5 \AA$. In this case, the value of $d$ is higher than that of $L$, and lower than that of $2 L$. This indicates that $1 / E t-7-O 4$ formed a partial bilayer structure in the smectic A phase. In this structure, the neighboring mesogenic groups overlap each other and the ammonium ions aggregate to form a sublayer in the smectic layer. The existence of the hydrophilic sublayer led to the formation of the partial bilayer structure that is different from the monolayer structure of Br-7. The $d$ values of $\mathrm{I} / \mathrm{Et}-3-\mathrm{O} 4$ and $\mathrm{I} / \mathrm{Et}-11$ O4 are 38.3 and $46.0 \AA$, respectively, while the $L$ values are 25.7 and $35.3 \AA$, respectively. It is believed that $\mathrm{I} / \mathrm{Et}-3-\mathrm{O} 4$ also formed the same bilayer structure as that of I/Et-7-O4 (Figure 4). In the case of I/Et-11O4, the mesogenic cores overlap each other (Figure 5). 


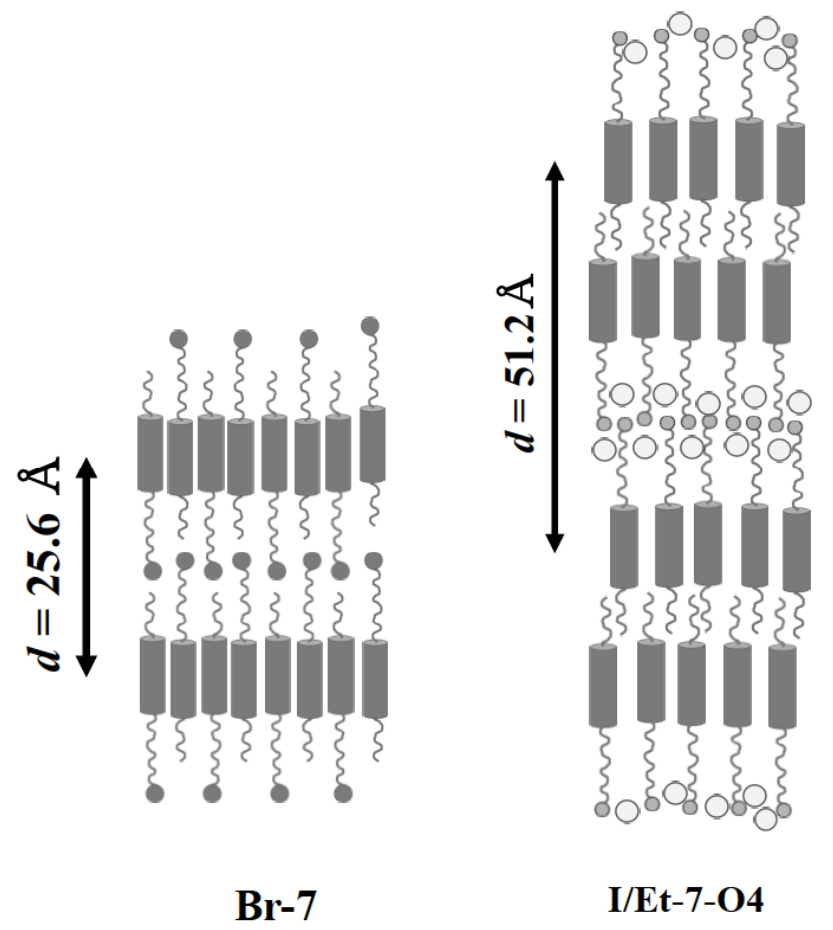

Figure 4: Possible packing models of $\mathrm{Br}-7$ and $\mathrm{l} / \mathrm{Et}-7-\mathrm{O} 4$.

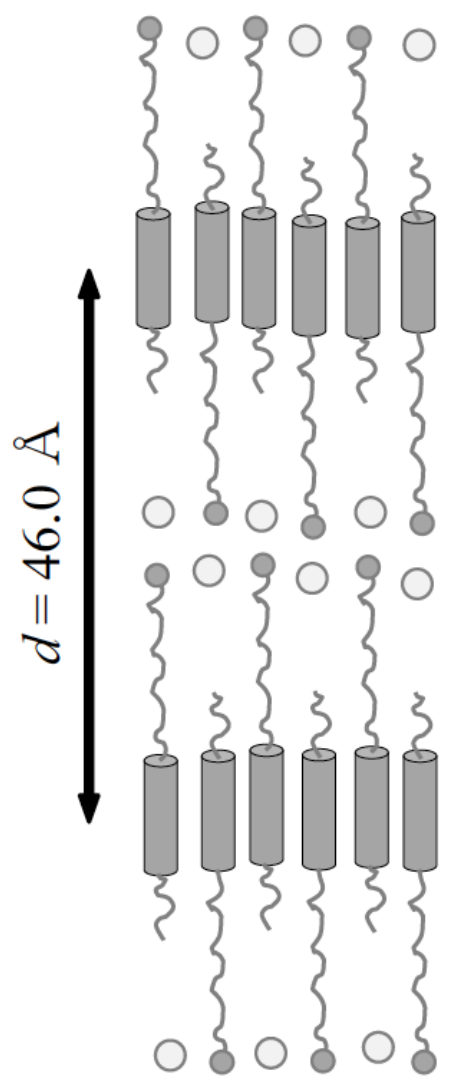

Figure 5: Possible packing model of I/Et-11-O4.

The nonionic family Et- $n-\mathrm{O} 4$ did not exhibit the liquid crystalline phase. However, the ionic family l/Et-n-O4 formed an enantiotropic smectic $A$ phase with enhanced thermal stability. This is due to the presence of ionic interactions. The ionic interactions resulted in the formation of the smectic layer structure consisting of ionic and nonionic sublayers. Furthermore, the alignment of the mesogenic groups was stabilized by the ionic sublayers.

\section{CONCLUSIONS}

The ILCMs (I/Et- $n$-O4) with the butoxy terminal group were found to exhibit the smectic A phase whereas, the nonionic family of $\mathrm{l} / \mathrm{Et}-n-\mathrm{O} 4$ did not exhibit the liquid-crystalline phase. This result indicates that the formation of the smectic $A$ phase was due to the ionic interactions between the ammonium cations and the iodide counterions. It is considered that the smectic A layer of $\mathrm{l} / \mathrm{Et}-\mathrm{n}-\mathrm{O} 4$ consisted of hydrophilic and hydrophobic sublayers. The hydrophilic sublayer was formed through the aggregation of ionic and hydroxyl groups. The formation of the hydrophilic sublayer contributes effectively to the formation of the smectic $A$ phase.

I/Et-7-O4 exhibited the widest temperature of the smectic $A$ phase and the highest phase transition temperatures among the I/Et- $n-\mathrm{O} 4$. Consequently, the thermal stability of the SmA phase increases in the order of 1/Et-3-O4, I/Et-11-O4, I/Et-6-O4, I/Et-7-O4. This means that the thermal stability of the smectic $A$ phase is controlled by the length of the spacer which connects the mesogenic group with the ionic group.

\section{REFERENCES}

[1] Collings PJ, Patel JS. Handbook of Liquid Crystal Research New York: Oxford University Press 1997; 329-331.

[2] Wu J, Nata M, Ujiie S. Anisotropic properties of mesogenic surfactants with ionic liquid core. J Appl Sol Chem Model 2014; 3: 8-14. https://doi.org/10.6000/1929-5030.2014.03.01.2

[3] Wu J, Ujiie S. Ionic liquid crystalline materials exhibiting smectic C phase. Mol Cryst Liq Cryst 2012; 563: 67-74. https://doi.org/10.1080/15421406.2012.688617

[4] Tschierske C. Micro-segregation, molecular shape and molecular topology - partners for the design of liquid crystalline materials with complex mesophase morphologies. J Mater Chem 2001; 11: 2647-71. https://doi.org/10.1039/b102914m

[5] Binnemans K. Ionic liquid crystals. Chem Rev 2005; 105: 4148-204. https://doi.org/10.1021/cr0400919

[6] Ujiie S, limura K. Thermal properties and orientational behavior of a liquid-crystalline ion complex polymer. Macromolecules 1992; 25: 3174-8. https://doi.org/10.1021/ma00038a024

[7] Ujiie S, limura K. Formation of smectic orientational order in an ionic thermotropic liquid-crystalline side-chain polymer. Polym J 1993; 25: 347-54.

https://doi.org/10.1295/polymj.25.347 
[8] Wu J, Ujiie S. Synthesis and liquid crystal formation of ionic polymers having hydrophobic side-chains. Sen'l Gakkaishi 2013; 69: 34-38

https://doi.org/10.2115/fiber.69.34
[9] Ujiie S, limura K. Ammonium halide type thermotropic liquidcrystalline polyethyleneimines and those low-mass model compounds. Chem Lett 1990: 19: 995-8. https://doi.org/10.1246/cl.1990.995 